\title{
Téoros
}

Revue de recherche en tourisme

\section{Les représentations du paysage et l'attractivité touristique}

Le cas « Tremblant » dans les Laurentides

\section{Fabienne Joliet et Thibault Martin}

Volume 26, numéro 2, été 2007

L’attractivité touristique des territoires

URI : https://id.erudit.org/iderudit/1070947ar

DOI : https://doi.org/10.7202/1070947ar

Aller au sommaire du numéro

Éditeur(s)

Université du Québec à Montréal

ISSN

0712-8657 (imprimé)

1923-2705 (numérique)

Découvrir la revue

Citer cet article

Joliet, F. \& Martin, T. (2007). Les représentations du paysage et l'attractivité touristique : le cas « Tremblant » dans les Laurentides. Téoros, 26(2), 53-58. https://doi.org/10.7202/1070947ar d'utilisation que vous pouvez consulter en ligne. 


\section{Les représentations du paysage et l'attractivité touristique Le cas «Tremblant» dans les Laurentides ${ }^{1}$}

\section{Fabienne Joliet et Thibault Martin}

À présent, c'était le Tremblant (le lac) qui ouvrait devant elle son énorme livre à la page illisible et qui ne se souciait pas d'être lue, dans l'épaisse reliure de la forêt. Il ne se prêtait pas volontiers. Il fallait lui demander la permission de regarder ses images.

Marie Le Franc, Hélier, fils des bois, p. 53.

Le mont Tremblant, situé dans les Laurentides au Québec, est une des destinations touristiques parmi les plus attractives du Canada, voire de l'Amérique du Nord, tant pour les vertus de son paysage "naturel » que ses qualités récréatives. Sa trajectoire paysagère connaît des étapes et des modes de mise en valeur successifs depuis le milieu du XIXe siècle jusqu'au développement récent de la plus importante station de ski de l'est de l'Amérique du nord. Dans cet espace temps d'environ 150 ans, le génie des lieux s'avère aujourd'hui schizophrénique : aux pieds du mont Tremblant légendaire un lac majestueux avec, dans sa partie nord, une forêt "préservée » et, dans sa partie sud, une mise en scène exacerbée.

Les représentations du paysage du lac Tremblant - qui vont nous intéresser ici permettent de passer de l'autre coté du miroir, de comprendre quelles sélections du regard et des pratiques sont opérées sur ce territoire en termes d'attractivité. Ainsi, avec le parti pris de la réalité perçue et vécue, et vu de la fenêtre de LacTremblant-Nord, cet article propose d'appréhender comment le filtre des représentations du paysage joue sur la réalité géo-



La nature comme paysage vierge. Vue panoramique du nord-ouest du lac : au second plan la baie des Chevreuils, les îles aux Cèdres, Leblanc et Laviolette et, au troisième plan, le mont Tremblant et les baies sud du lac.

Source : Peinture, Lac Tremblant Nord, 2004, M. Normandeau.

graphique et notamment quel type de naturalité les résidants de cette municipalité privilégient en choisissant de quitter le train en marche du développement de l'autre municipalité.

Dans un premier temps la relation qui unit représentations paysagères, modes d'appropriation et attractivité touristique sera abordée par le biais de l'étude de cas de Lac-Tremblant-Nord; puis, dans un second temps, l'expression de ce paysage ambivalent sera caractérisée, notamment par une mise en abîme de la naturalité "authentique » de Lac-Tremblant-Nord au regard de celle de la station Tremblant.

\section{Positionnement théorique et méthodologique : l'étude de cas Lac-Tremblant-Nord}

\section{Lac Tremblant: une étude de cas exemplaire}

L'étude de cas du lac Tremblant est exemplaire en termes d'attractivité, tant par le point focal touristique d'une région qu'il est devenu que par les représentations qu'il génère depuis plusieurs décennies. Le lac Tremblant est au cœur des Laurentides, région située au nord de la ville de Montréal et associée dans l'imaginaire québécois aux «Pays d'en haut », symboles de l'histoi- 
re sociale des Canadiens français et aujourd'hui lieu où s'enracine la construction sociale que les Québécois font de la nature.

Le lac Tremblant cumule deux superlatifs dans ses atouts géographiques: il est un des plus grands de la région avec une envergure de 15 kilomètres de long et il est surplombé par le sommet le plus élevé de la région, le mont Tremblant, qui atteint presque 1000 mètres d'altitude. Les structures touristiques développées sont parmi les plus anciennes dans les Laurentides (Beaudet, 1996). Le désenclavement par l'autoroute 15 et l'aéroport international de La Macaza témoignent de la saillance géographique de ce site au Canada, le propulsant dans un espace-temps de proximité quasi immédiate en Amérique du Nord, à une heure et demie de Montréal, deux heures d'Ottawa et quelques heures de Toronto et de New York.

Si ses qualités et sa localisation géographique sont devenues privilégiées, au sens propre du terme une "destination", son paysage spécifique incarne de plus un archétype paysager et social québécois. D'abord paysager, parce que les vallées, les lacs (4000 environ) et les montagnes faites de cette roche sombre du bouclier canadien qui affleure en de nombreux endroits - le tout couvert par une forêt dense dont les couleurs automnales exercent un attrait magique - constituent ce qui, dans l'imaginaire québécois, est la nature de ce pays par excellence. Ensuite social, parce que la région a été colonisée par des Canadiens français entraînés hors de Montréal par un clergé catholique en quête de territoires exempts de l'influence anglo-protestante.

Le genius loci du site de Tremblant réside donc, d'une part, tout particulièrement dans l'ampleur du lac, serti dans un écrin de forêt "boréale", dont la canopée se déroule sur l'échine de collines qui déferlent en houle à l'infini, avec le mythique mont Tremblant pour amer. La composition du paysage de Tremblant se présente effectivement de manière très homogène et de facto très clairement lisible et identifiable ${ }^{2}$. Enfin, à l'homogénéité du paysage s'ajoutent deux facteurs qui contribuent également à son appréciation: l'échelle et le mythe. L'échelle de l'infini marqué par la continuité tout autant que la démesure de la forêt et de la topographie confère une ampleur - pour ne pas dire une grandeur unique au paysage. À cela s'ajoute la dimension mythique du mont Tremblant, qui participe également de la puissance évocatrice de ce paysage. Mont «Tremblant», une dénomination issue d'une légende amérindienne selon laquelle la montagne gronde lorsque les hommes portent atteinte à la nature. Cet animisme des Amérindiens algonquins confère un acte double d'authenticité et de personnification (d'anthropomorphisme) pour le visiteur européen; ce qui n'est pas anodin quant à la construction identitaire d'un paysage et au sentiment d'appartenance des sociétés qui lui est associé.

Le genius loci du lac Tremblant réside d'autre part dans un archétype de nature québécoise construite socio-culturellement entre revendications francophones et anglophones. En effet, le clergé catholique rêvait de construire, depuis le milieu du XIX ${ }^{e}$ siècle, un Canada francophone et catholique, parallèle à celui des anglophones protestants. Cet autre Canada devait se développer au nord des zones de colonisation anglaises afin, comme l'exprimait le curé Antoine Labelle, figure emblématique de ce mouvement et acteur central du développement des Laurentides, "[qu'] en nous emparant du sol, depuis la vallée de l'Outaouais jusqu'à Winnipeg, nous empêch[i]ons qu'on nous passe sur le dos pour aller à la Baie d'Hudson » (cité par Potvin, 2003 : 41).

Le développement des Laurentides que l'on a longtemps appelé le "Nord» - on continue encore de le faire - n'était en somme qu'une composante de ce projet épique de maintenir une présence catholique et « française" forte au Canada que les ancêtres des Canadiens français avaient perdu sur les plaines d'Abraham (Morissoneau, 1978). Toutefois, le projet de colonisation des Laurentides a été accéléré par deux événements: tout d'abord l'arrivée, dans les années 1860, de colons écossais, suivie de celle de compagnies forestières anglosaxonnes, ce qui fit dire au même curé Labelle: "Voilà l'avenir de mes compatriotes en péril! Les protestants ne leur prendront pas ces bonnes terres! II est urgent de contrer leur avance en occupant, avant eux, ces belles plaines le long de la rivière Rouge, vers le Nord-Ouest. " (Cité par Potvin, 2003: 41.) Par ailleurs, des conditions économiques défavorables poussaient des centaines de familles de Canadiens français à s'expatrier en Nouvelle-Angleterre pour y trouver du travail dans les manufactures, ce qui a suffi à convaincre cet ardent défenseur des valeurs (famille, Église, et ruralité) au cœur de la culture des Canadiens français à activer le développement du "Nord", non seulement en ouvrant de nouvelles paroisses, mais aussi en y apportant les moyens de communication modernes, les routes et le chemin de fer.

Rien d'étonnant donc à ce que les caractères de ce paysage emblématique du Québec, mais aussi de l'est de l'Amérique du Nord, aient suscité depuis le début de sa trajectoire des aspirations de nature passionnée. Ainsi, des formes de naturalités différentes se sont structurées spatialement de part et d'autre du lac Tremblant. Par conséquent, ce sont les conditions géographiques et socio-historiques, les caractères de son paysage identitaire et les différentes naturalités qu'il abrite qui font l'exemplarité de Tremblant comme étude de cas au titre de la sélection qui s'opère sur le territoire selon des préférences, qui elles-mêmes engendrent réciproquement des comportements et des aménagements qui confortent ces distinctions. Cette "émergence paysagère » (Domon et al., 2000) sera abordée sous l'angle des représentations du paysage, qui sont des révélateurs privilégiés de la forme d'appréciation accordée aux paysages et à la nature.

\section{Les représentations du paysage / l'attractivité touristique}

Qu'ont en commun les représentations du paysage et l'attractivité touristique? Une sensibilité hédoniste qui opère des sélections dans le paysage et l'espace. Dans les lignes qui suivent, ces deux notions feront l'objet d'une brève définition avant d'être croisées.

Les représentations du paysage constituent tout d'abord la matrice esthétique qui régit notre appréciation de celui-ci. En effet, la perception d'un paysage n'est autre que le fonctionnement optique de notre cerveau combiné aux images socioculturelles qui s'y impriment au cours de l'expérience du monde qui nous entoure. Ces images, avant d'être stockées dans le cerveau, sont essentiellement produites par l'art; elles instaurent des prises de vues particulières et des lieux de prédilection à l'origine d'une attraction sélective au sein d'un même paysage, en tant que ces derniers coïncident 
plus ou moins consciemment avec les archétypes enregistrés. Ces paysages "montrés", localisés, et de fait projetés et attendus, cristallisent des images références et des zones de sensibilité paysagères qui apparaissent comme en surbrillance sur l'écran des paysages de l'« ordinaire».

Quant à l'attractivité touristique, elle peut être définie comme la ou les zones d'un périmètre destiné aux loisirs qui font l'objet d'une attirance plus forte que d'autres, induisant une concentration de fréquentation en ces points. Cette concentration des attraits peut être due à la disposition d'équipements, leur qualité ou encore au cadre paysager de ces derniers. Car force est de constater que l'espace n'est isotrope que sur la carte et que sa réalité - à savoir celle que lui attribuent les sociétés qui le vivent et a fortiori les populations touristiques, qui, dans leur recherche de délocalisation, ont une attente de plaisir relativement précise et forte - est tout autant qualitative.

En l'occurrence, les représentations du paysage créent des modèles polarisants qui deviennent ensuite des canons touristiques polarisés à consommer. Et plus la consommation est élevée, plus les représentations sont nombreuses et prégnantes. II faut avoir vu, il faut y être allé! C'est en synergie que ces deux notions créent donc un système de distinction de territoires. L'analyse des représentations du paysage proposée ici permettra ainsi d'envisager quels archétypes sont en vigueur et quels filtres soustendent les deux modes de naturalité qui se sont développés au nord et au sud du lac Tremblant. Cette analyse des représentations répond à une méthodologie spécifique dont les éléments essentiels sont exposés dans la section qui suit.

\section{L'analyse des représentations et l'analyse de discours, ou l'image et les mots pour le dire}

Les représentations du paysage sont appréhendées par le biais de deux types de données complémentaires, à savoir les images paysages, non verbales, auxquelles s'ajoutent celles, verbales, qui produisent ou révèlent des images mentales de paysages (entretiens - verbal oral ; et littérature verbal écrit).

Le corpus iconographique rassemblé ${ }^{3}$ comprend 46 représentations du territoire de
Tremblant, de 1932 à nos jours. Les figurations sont picturales ou photographiques (sous la forme de tableaux et de cartes postales). Elles répondent à des critères de sélection: elles sont ciblées sur les thématiques du paysage du lac et/ou du mont Tremblant, des deux villages dits de Tremblant - l'ancien et la station - ou encore de l'hôtellerie et de l'activité sportive. Toutes ces représentations iconographiques sont inventoriées dans un "carnet ", permettant le traitement des données, mais donnant aussi la possibilité de les soumettre à consultation lors des entretiens.

Afin de compléter ces données de l'imagerie paysage, deux autres types de données produisant de l'image paysage par les mots sont investiguées: les entretiens et la production littéraire. Huit entretiens ont été conduits avec des résidants de Lac-Tremblant-Nord pour interpréter le paysage vécu, l'expression de la naturalité autour du lac. II s'agit d'entretiens ouverts réalisés chez l'habitant, accompagnés de la consultation de leur album de photos de famille, de la visite de la propriété, puis du recueil de réactions sur le carnet iconographique. L'échantillon est constitué de trois résidants à l'année, vivant dans l'ancien village de Tremblant ou à Sainte-Agathe-des-Monts, et de cinq propriétaires et résidants riverains saisonniers, dont deux familles francophones, deux anglophones et une mixte, de provenance urbaine (Montréal, Toronto).

Quant à la production littéraire, elle se résume à une œuvre: le roman de Marie Le Franc, Hélier, fils des bois, qui se déroule sur le territoire de Lac-TremblantNord. Cet ouvrage de 1937 est unique, tant par le témoignage historique et géographique des lieux qu'il offre que par la fiction paysagère qu'il restitue sous la plume de Marie Le Franc, préfigurant déjà du dualisme entre deux naturalités, divisant le nord et le sud du lac.

\section{Comment traiter ces données? Quels construits du territoire et quelles apparitions paysagères expriment-elles?}

L'analyse des représentations est faite dans le but de répondre aux deux questions précédentes. Elle porte sur deux niveaux interdépendants: un premier niveau d'analyse géographique de la prise de vue sélectionnée (qui questionne les points de vue et les cônes visuels: d'où est prise la vue et quelle partie du paysage elle montre) et un second niveau d'analyse de composition esthétique de cette vue (qui s'intéresse à la construction de l'image pour représenter tel paysage et interroge les marqueurs identitaires).

Afin d'envisager la trajectoire de ce paysage «naturel d dans l'espace et dans le temps, la méthodologie (Joliet, 2004) répond à l'identification des lieux et des thématiques de nature qui transparaissent des ces trois types de données (l'image, les entretiens et la littérature). Elles sont croisées et comparées, additionnées et synthétisées. L'étude de Lac-TremblantNord est par conséquent exemplaire quant à l'appréhension de la question de l'attractivité touristique de sites "naturels». Qui plus est, elle est envisagée au travers du filtre paysager socioculturel de LacTremblant-Nord, môle de résistance fidèle à une image traditionnelle face au développement de la station récréative. Les résultats de l'analyse révèlent une expression de nature « authentique » versus celle "construite» de la station de village Tremblant.

\section{Résultats}

\section{Un lac, deux naturalités}

Le géosystème de Lac-Tremblant-Nord est caractérisé par une nature volontairement peu socialisée, résultat de la conjonction entre un habitat ancien et discret de cabanes à l'interface lac-forêt ou " cottages " (Le Franc, 1937) et d'une nouvelle implantation de demeures prestigieuses contemporaines qui s'inscrivent en "balcon"sur les hauteurs du lac, et non derrière un rideau d'arbres comme la précédente génération riveraine. De plus, ce géosystème est aussi tributaire du clivage socioculturel qui prévaut au Québec, puisque les dispositifs anglophone et francophone diffèrent sensiblement: la cabane, les toilettes sèches font davantage partie de l'habitat des francophones, alors que le ponton, le garage à bateaux et la "cabane à dormir » se retrouvent davantage chez les anglophones. Coté architecture, la cabane en rondin typiquement francophone coexiste avec le style Nouvelle-Angleterre, marqué notamment par la présence d'une galerie. 
La dynamique historique de colonisation par les Canadiens français de ces terres s'est développée selon le principe du rang qui prévalait dans la vallée du SaintLaurent, découpage foncier déterminé ici par l'accès au lac, qui a produit des parcelles tantôt vastes, approximativement carrées de $200 \times 200$ ou 300 pieds de côté, tantôt plus profondes et rectangulaires, en "lame de parquet». Quoi qu'il en soit, chacune a fait l'objet d'une "patente ", attestant d'un travail de défrichement suivi d'un engagement de résidence ${ }^{4}$.

Coté fonctionnalité et pratiques du territoire, Lac-Tremblant-Nord est vécu sous la forme d'une activité forestière révolue et des expériences de loisirs rituelles. Le parc national, adjacent aux parcelles de LacTremblant-Nord, est I'héritier de la réserve forestière de 1895. La mise en place de cette réserve atteste de la vocation peu récréative du territoire lors de son « ouverture ", dont la fonctionnalité était essentiellement tournée vers l'exploitation du bois (par la Canadian International Paper / CIP entre autres). Contrairement à d'autres contrées forestières voisines, on trouvait peu (une vingtaine seulement) de pourvoiries dans le territoire du parc (Fournier, 1981). Longtemps, le lac a été associé à cette activité industrielle et a servi au transport par flottage des troncs, acheminés du nord au sud par la rivière Cachée. Aujourd'hui, bien que les activités industrielles aient disparu, le lac demeure encore «traditionnel », si bien que plusieurs activités de loisir pratiquées dans cette nature, notamment la chasse, la pêche ou le bain rituel dans le lac, attestent de l'ancrage historique des pratiques sociales des riverains. On y trouve un "traditionalisme » sans folklore, un sentiment d'appartenance qui se traduit aussi par une forte transmission intrafamiliale des propriétés par héritage, se démultipliant même afin de permettre aux différents descendants de bâtir sur le terrain-territoire familial et de l'écrire sur la page des lieux.

En revanche, face à cette "permanence", les modes de locomotion traditionnels, canot l'été et raquettes l'hiver, sont remplacés aujourd'hui par des embarcations motorisées quand le lac est praticable ainsi que par les motoneiges et les quatre-roues. De même, la voile et la régate annuelle trouvent de plus en plus d'amateurs. Signe d'une transformation sociale et d'un affai- blissement des réseaux traditionnels dus aux ventes et aux transferts de propriété, les gens qui y assistent se connaissent de moins en moins, ce qui fait que cet événement contribue à institutionnaliser un nouveau mode de sociabilité qui n'est plus uniquement inscrit dans des relations de proximité, mais aussi dans un goût partagé pour une activité de loisir de « distinction ».

Par conséquent, cette nature, discrètement aménagée et appropriée par quelques pratiques traditionnelles maintenues malgré les transformations sociales, est le fruit d'une sensibilité dont témoignent les résultats de l'étude des représentations de son paysage «naturel ». Le sentiment de nature inhérent à ce géosystème est porté par une narrativité profonde, discrète, qui se veut une vision authentique répondant à des pratiques initiatiques; une narrativité rendue explicite par les types de données analysées, les représentations iconographiques, le discours des entretiens et du roman de Marie Le Franc. La naturalité qui sourd des trois sources de cette analyse décline plusieurs thématiques, telles que l'expérience (De Blois Martin, 1999), le recueillement, la vie physique et familiale au sein d'une nature originelle souveraine, grande, métaphorique d'un état d'être.

Un premier constat de ces résultats tient au nombre faible de représentations iconographiques du paysage. Cette rareté est un résultat en soi, qui témoigne d'une discrétion, d'un relatif isolement volontairement entretenu, d'une volonté de ne pas dévoiler, de limiter toute attractivité extérieure; cela, malgré une ouverture récente de son paysage au regard d'autrui, rendue possible par des accès physiques et donc des ouvertures visuelles panoramiques dues aux nouveaux lotissements (La Tranquillité et La Sérénité) au sommet nord-ouest du lac, accessibles par une route, alors qu'auparavant l'habitat était confiné au bord du lac sans autre accès.

En dépit du faible nombre de représentations iconographiques de cette partie nord du paysage du lac (soit 16 sur Lac-TremblantNord et 33 sur la partie sud du lac, village Tremblant), leur analyse qualitative permet un deuxième constat, de remarquer la non-figuration de l'habitat de manière systématique, bien que ce dernier soit présent et fondu dans la nature. Cela est susceptible d'attester d'une volonté plus ou moins consciente

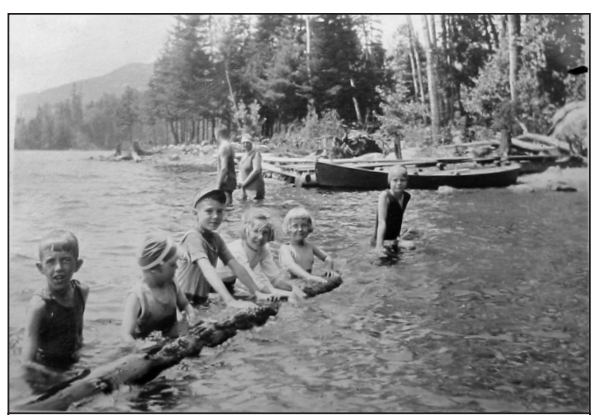

La nature comme écrin d'une vie authentique. Source : Photo de famille, album Rawley, années 1950.

d'effacer les cabanes de la dimension « naturelle» des lieux. Toujours sur ce plan de la construction esthétique de la composition, le paysage de la partie nord apparaît comme représenté à partir d'une vision " centripète " puis "centrifuge » à l'échelle d'un siècle: les images produites jusqu'aux années 1970 intégraient généralement le lac comme premier plan intimiste donnant à voir, vers l'extérieur, les second et troisième plans de la forêt et des montagnes environnantes; tandis que depuis, à l'inverse, les représentations sont plutôt dimensionnées en format "grand angle ", orientées des sommets vers le lac, permettant d'offrir un panorama.

Enfin, troisième constat, chromatique, ce sont les couleurs de l'automne et de l'hiver qui transparaissent comme source d'inspiration privilégiée de ce paysage.

Au niveau de l'analyse quantitative des œuvres en fonction de leur répartition géographique sur le territoire, les résultats montrent que le corpus met en surbrillance certains lieux par rapport à d'autres. Elles privilégient notamment, jusqu'aux années 1970, la rivière Cachée et son embouchure et la baie des Chevreuils, tandis que les représentations contemporaines ${ }^{5}$ (> 2000) emblématisent également la confluence de la rivière Cachée de la rive tout en amorçant la découverte esthétique de la ligne de crête dite du « Nez de l'indien » sur la rive ouest et enfin un point de vue panoramique pris du nordouest du lac en surplomb de la falaise de La Palissade vers le mont Tremblant en fond de scène. Cette récente construction paysagère met en évidence l'escarpement du cadre lacustre au premier plan, les anses découpées de la rive ouest - baie des Chevreuils, baie des Ours - ainsi que les îles - aux Cèdres, Leblanc et Laviolette - au deuxième plan et le mont Tremblant à l'arrière-plan. La baie des Ours, baie septentrionale du lac, ne figure jamais cependant... 
La narrativité portée par cette sélection du territoire «naturel » de Lac-Tremblant-Nord est affinée par les données verbales recueillies. L'interprétation des huit entretiens présente en effet le paysage du nord du lac plus comme un lieu de l'enracinement familial ou individuel que comme un paysage : un marqueur identitaire fort, expression des deux ou trois générations qui ont succédé aux parents pionniers de cette villégiature depuis le début du XXe siècle. La consultation des albums photographiques de famille montre également que le paysage est peu représenté pour lui-même, celui que l'on pourrait regarder à la maison en semaine à Montréal ou à Toronto pour entretenir le vécu rituel des fins de semaines ou des saisons d'été est quasi absent de ces albums. $Y$ figurent quand même quelques scènes paysagères contemplatives, comme une photo représentant une femme de dos devant un lac lointain ainsi qu'une autre immortalisant les jeux de bain pour enfants.

Quant à elle, la représentation écrite du roman de Marie Le Franc, Hélier, Fils des bois, est au contraire totalement imbriquée au paysage, (uniquement en saison d'estive) qui lui sert de toile de fond métaphorique, mais aussi comme protagoniste du roman. L'auteure utilise quatre procédés, quatre idées-forces pour représenter la naturalité vécue sur la rive nord du lac Tremblant au début du XXe siècle. Tout d'abord elle opère une personnification de la nature en lui conférant une animalité vibrante: "La forêt entière se concentrait autour du lac. Elle avait attendu que le jour fût complètement tombé pour venir en rampant s'y abreuver " (Le Franc, 1937 : 51), ou encore « apaisé, le lac se couchait en rond pour reprendre son

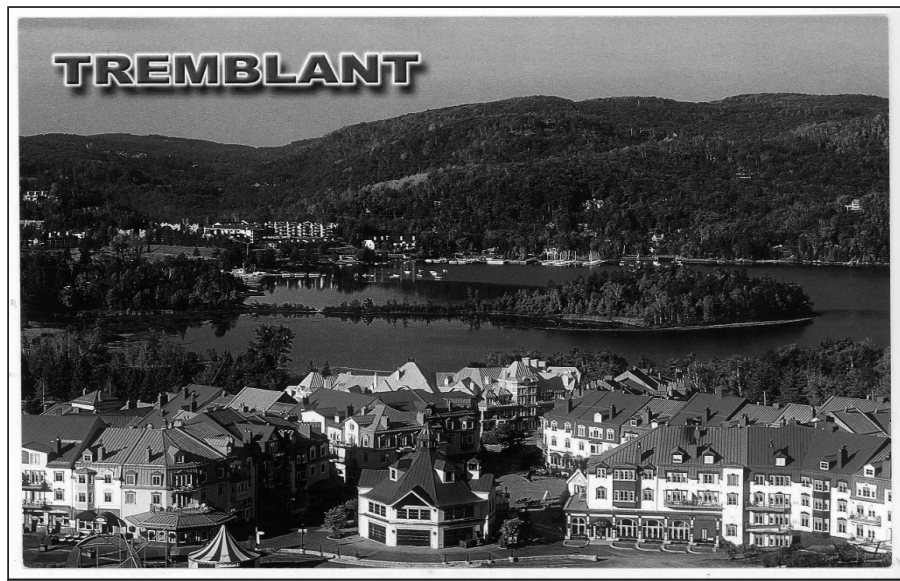

Carte postale «Tremblant, la première station de villégiature de l'Est canadien ». Photo: P. Quitemelle, collection personnelle de l'auteure. sommeil » (idem: 52). Ensuite, la nature est isolement et retranchement: "Julienne Javilliers fut aussi séparée du monde que sur une île perdue dans les mers lointaines. Le mot solitude paraissait faible, appliqué à ces lieux »(p. 49) ou bien « une paix d'éternité d'où on n'entendait plus battre le cœur du monde » (p. 243). Troisième pivot autour duquel s'articule la construction de Marie Le Franc : l'infini et l'éternité : «Le Tremblant comptait par siècles, non par heures » (p. 92) « Jusqu'où vont les montagnes? demanda-t-elle. Mais jusqu'au bout... lui répondit-il. » (p. 70) Comme dernier procédé, elle associe la nature originelle avec l'être profond (p. 231) : « II se sentait tissé dans la trame lumineuse des choses. [...] Hélier avait sa façon à lui de célébrer le dimanche. II se passait d'église [...] II avait fallu se faire une religion à sa manière, s'agenouiller où l'on se trouvait, accepter pour vitraux les découpures du ciel à travers les branches ou les rosaces du givre sur les vitres. » (p. 78)

Les différentes représentations du paysage de Lac-Tremblant-Nord évoquées ici convergent donc vers le témoignage d'une nature perçue comme authentique au sens de «vraie». Elle est authentique parce qu'elle est vécue comme vivante, "éternelle» et « infinie » et parce qu'elle permet à l'homme de s'épanouir par une spiritualité spontanée et non pas institutionnalisée.

Cela étant dit, si cette nature au nord du lac donne lieu à peu de représentations iconographiques (photos, dessins, peintures), on peut néanmoins souligner le fait que les tableaux du Groupe des Sept ont un impact et une puissance évocatrice sans doute aussi forte que la production abondante de cartes postales sur la partie sud du lac. On peut également émettre I'hypothèse que cette faible représentation pourrait être due au caractère approprié de ce territoire que l'on veut garder privé pour (se) le préserver. De surcroît, le nombre de représentations qui évolue peu dans le temps atteste que cette nature, réservée à un petit nombre, doit rester confidentielle, ce qui implique que toute représentation ostentatoire est à éviter.

\section{Nature " authentique " versus nature " construite»}

Cette nature authentique produite par les résidants du lac Tremblant n'est certes pas unique. Les autres acteurs du territoire environnant le mont Tremblant, notamment la station de ski, ont quant à eux produit à la fois en parallèle et en opposition une version prête à consommer de cette même nature. C'est ainsi que le "capital paysage ", selon la formule de Domon, Beaudet et Joly (2000), du site de Tremblant se décline, aujourd'hui, en deux narrativités et temporalités distinctes ${ }^{6}$.

Dans la première narrativité, celle des résidants du lac Tremblant, la représentation du territoire est discrète car l'existence de cette nature encore authentiquement vraie doit être tenue secrète afin d'être préservée. Par contre, du côté de la seconde narrativité, celle de la station touristique, la nature doit au contraire être mise en scène afin que son authenticité soit connue de tous. C'est ainsi que la mise en marché du mont Tremblant se fait elle aussi au nom de l'authenticité, celle-ci s'articulant autour de deux pivots. Le premier est bien sûr le caractère vierge et infini de la nature, le second met au contraire l'accent sur la dimension socialisée de celle-ci. Cet élément est d'ailleurs de plus en plus prégnant dans les représentations de la station touristique qui utilise l'héritage culturel québécois, c'est-à-dire les vieux villages de Mont-Tremblant et de Saint-Jovite fondés au début du XXe siècle par des pionniers canadiens-français, comme cadre de référence historique justifiant la mise en scène grandiose de la station elle-même, actualisation exacerbée du style architectural québécois traditionnel.

II est sociologiquement remarquable que chacun de ces modèles de représentation soit associé à une vision spécifique de l'aménagement social du territoire. En effet, le repli sur soi de Lac-Tremblant-Nord s'oppose à l'ouverture sur l'extérieur qui caractérise le site de Mont-Tremblant, y compris le parc qui est non riverain du lac mais adjacent. Le désir de partage est important à relever parce qu'il procède d'un projet démocratique très canadien qui consiste à vouloir mettre la nature à la portée de tous. En effet, les parcs nationaux du Canada ont été créés à partir de la fin du XIXe siècle à la fois pour protéger les sites patrimoniaux et pour permettre au grand public d'y avoir accès. Aujourd'hui 
certains de ces parcs - Banff étant le premier et le plus connu d'entre eux - sont devenus des destinations qui attirent, du fait de la mise en scène grandiose de la nature qu'ils opèrent, un tourisme de masse. Tremblant avec son parc d'un côté et sa station touristique internationale de l'autre s'inscrit donc dans cette tradition canadienne de démocratisation de la nature. Lac-Tremblant-Nord avec sa fermeture et son désir de ne pas partager avec la masse son territoire s'inscrit par ailleurs dans l'habitus de l'élite économique nord-américaine qui abhorre la mixité sociale et n'hésite pas à entourer de "murs » (ou de mesures démotivantes rédhibitoires) les quartiers où elle réside afin de se protéger du regard et de la compagnie de ceux qui ne partagent pas ses valeurs.

Certes, les riverains du lac Tremblant n'ont peut-être pas entouré de palissades physiques leur territoire, mais ils ont quand même réussi à l'exclure du processus de restructuration égalitaire du territoire que le gouvernement du Québec avait voulu imposer à la région. En effet, en 1999, dans le but de rassembler le patchwork social et de faire bénéficier tous les résidants du "grand Tremblant» de l'effet «locomotive» de la station récréative ${ }^{7}$, le gouvernement provincial imposa une fusion municipale d'où allait naître la municipalité de Mont-Tremblant composée de Saint-Jovite paroisse, SaintJovite ville, Lac-Tremblant-Nord et MontTremblant (comprenant le village historique et la station) ${ }^{8}$. Toutefois, les quelques deux cents résidants de Lac-Tremblant-Nord qui s'étaient toujours opposés à cette fusion ont réussi en 2006 à se séparer de la nouvelle entité municipale pour retrouver leur indépendance sur le plan de l'urbanisme (tout en conservant la communauté des autres services, l'enlèvement des ordures par exemple). Cet échec de l'action publique destinée à créer une solidarité territoriale transcendant les sentiments d'appartenance et les conflits de classe a échoué parce qu'elle n'avait pas tenu compte que si «l'action délibérée, publique par opposition au privé domestique [...] bâtit des solidarités territoriales. II faut ajouter l'action culturelle (le symbolique, les significations partagées) qui construit l'identification» (Morissoneau, 1997). En somme, l'opposition à la fusion municipale n'était pas économique, mais bien identitaire et cette identité forte des résidants de LacTremblant-Nord s'incarne et se révèle à travers les représentations qu'ils donnent de leur territoire.

\section{Conclusion}

Archétype paysager et socioculturel québécois, le lac Tremblant se décompose en un "système» ternaire de naturalités distinctes: le nord du lac, le sud du lac ainsi que l'est (le parc national), qui, sans être riverain du lac, participe de la demande sociale de nature en offrant une alternative préservée et publique.

On observe que Lac-Tremblant-Nord s'est retranché, en demeurant une vitrine impénétrable de nature emblématique, au paysage porté moins par les représentations iconographiques que par l'affect, c'est-à-dire les représentations verbales du discours des résidants et de la littérature. Une confiscation paysagère qui fait plus l'objet d'une acceptation que d'une résignation, car cette construction de nature «cabane-lac-forêt» est considérée comme une aspiration et un accomplissement du mode de vie canadien, «that we call home» (Jacobs, 2004).

Ainsi figure de palingénésie, le paysage de Lac-Tremblant-Nord se présente paradoxalement comme l'expression habitée d'une des grandes natures originelles de la planète, de l'Amérique française et anglaise, c'est-à-dire un des fondements de I'histoire du Québec, du Canada (Cros, 2007) qui lui a permis notamment de se distinguer de la vieille Europe dans ce que celle-ci n'avait pas.

Fabienne Joliet est maître de conférence au département paysage de I'Institut national d'horticulture d'Angers.

Thibault Martin est professeur au département de travail social et des sciences sociales à I'Université du Québec en Outaouais.

\section{Notes}

1 Nous tenons à remercier chaleureusement, Jean Grégoire, Louise Royer et les résidants du lac qui ont généreusement accepté d'être interviewés.

Fabienne Joliet remercie l'Ambassade du Canada pour la Bourse de Recherche qu'elle a obtenu lui permettant de sejourner à l'Université de Montréal et au Lac Tremblant en juillet 2006.

2 Cela étant, la nature des entités fondatrices (le lac, la forêt, les montagnes) engendre deux directions de perception privilégiées, orientées par le rapport intérieur / extérieur, plein / vide. Une direction, un « sens » de vues et d'accès, qui vont conditionner les pratiques et les aménagements dans ce paysage. II s'agit en effet d'un paysage à visibilité réduite de l'intérieur par l'opacité de la forêt qu'accusent le relief et donc les espaces masqués. À l'inverse, les ouvertures qu'offrent les lacs (les «vides" du dedans) et les sommets (les vues du dessus) constituent des percées visuelles extrêmement puissantes par contraste.

3 Sources: Bibliothèque nationale du Québec, Musée McCord et Musée des beaux-arts de Montréal, exposition ancienne gare de Tremblant, mairie de Tremblant, boutiques de la station Tremblant.

4 Le premier contrat d'achat date de 1902, par $M^{l l e}$ Stuart qui acquiert ainsi l'île Cachée (dénommée par la suite «île Stuart»).

5 Souvenir Folder - Lac Mercier, Québec, Canada, 1930.

6 L'opposition actuelle entre ces deux narrativités est le résultat d'un long processus de croissement, d'association et de dissociation de perspectives. La place manque ici pour développer cette question, mais elle donnera lieu à une autre publication.

7 II est utile de préciser que, malgré les retombées économiques importantes de la station de loisir du mont Tremblant (elle reçoit 2 millions de touristes par année), la petite ville de Saint-Jovite, centre historique et lieu d'encrage de la population locale, demeurait pauvre et ne profitait que peu des retombées de l'exploitation de son patrimoine environnemental.

8 Le projet de loi fut adopté le 10 novembre 1999 et le processus de fusion qui donna naissance à la nouvelle ville de MontTremblant (d'une population de 7500 habitants) fut complété le $1^{\text {er }}$ janvier 2001.

\section{Bibliographie}

Beaudet, G. (1996), "Un bref regard sur l'architecture de la villégiature et du tourisme", Téoros, vol. 15, no 1, p. 39-42.

Cros, I. (2006), « Nature, histoire et construction nationale au Canada: une étude des écrits de Harold Innis, Donald Creighton et Arthur Lower ", Études Canadiennes / Canadian Studies.

De Blois Martin, Ch. (1999), «L'expérience touristique, retrouver les liens échappés ", Actes du Colloque de l'Université rurale québécoise au Bas-Saint-Laurent, 4-8 octobre.

Domon G., G. Beaudet, et M. Joly, 2000. Évolution du territoire laurentidien, caractérisation et gestion des paysages, Université de Montréal, Chaire en paysage et environnement, $138 \mathrm{p}$.

Fournier, M. (1981), Histoire du parc du Mont Tremblant, Québec, Ministère du Loisir, 65 p.

Jacobs, P. 2004, « Folklore and Forest Fragments: Reading Contemporary Landscape Design ", Landscape Journal, vol. 2, n 2, p. 85-100.

Joliet, Fabienne (2004), « Quelle naturalité du paysage ligérien? La Loire du Maine-et-Loire", Noroîs, no 192/3, p. 85-94.

Le Franc, Marie 1937, Hélier, Fils des bois, Nelson, Paris, $288 \mathrm{p}$.

Morissoneau, Ch. (1978). "La colonisation équivoque ", Recherches sociographiques, vol. 2 , n० 19, p. 35-55.

Morissoneau, Ch. (1997), «La délibération territoriale contre l'appartenance élastique ", Communication au $65^{\mathrm{e}}$ Congrès de l'Association francophone pour le savoir (ACFAS).

Potvin, M. (2003), Mont-Tremblant, au cœur des Laurentides, Outremont, Éditions du Trécarré. 